\section{Evolução intergeracional da estatura no Estado de Pernambuco, Brasil, entre 1945 e 2006. 1 - aspectos descritivos}

\author{
Intergenerational evolution of stature in \\ Pernambuco State, Brazil (1945-2006). \\ 1 - descriptive aspects
}

José Natal Figueiroa 1

João Guilherme Bezerra Alves 1

Pedro Israel Cabral de Lira 2

Malaquias Batista Filho ${ }^{1}$

\footnotetext{
1 Instituto de Medicina Integral Professor Fernando Figueira, Recife, Brasil. 2 Universidade Federal de Pernambuco, Recife, Brasil.

Correspondência J. N. Figueiroa Instituto de Medicina Integral Professor Fernando Figueira. Rua dos Coelhos 300, Recife, PE 50070-550, Brasil. natal@imip.org.br
}

\begin{abstract}
Linear regression models with random intercepts were used to describe the evolution in stature among residents in Pernambuco State, Brazil, in 2006, born from 1945 onward. From 1947 to 1987, yearly height gain was $0.23 \mathrm{~cm}$ in adult men and $0.15 \mathrm{~cm}$ in women. In relation to World Health Organization standard values (2006), children and adolescents showed a declining annual height deficit of -0.019 z-scores (H/A) for girls and -0.013 for boys (1987-2006). Men and women who completed 19 years of age in 1987 showed a height deficit of $5.0 \mathrm{~cm}$. Projecting the trends observed in 2006, adult men and women in Pernambuco would wait 22 and 33 years, respectively, to reach the international standard. Within 6 to 7 years, children under five would show a height deficit of $2.3 \%$, which is the occurrence verified in the WHO standard, thus correcting the historical growth retardation in this population group in Pernambuco.
\end{abstract}

Anthropometry; Growth and Development; Stature by Age

\section{Introdução}

Em nível populacional, o crescimento humano, notadamente em seu parâmetro mais característico (a altura), expressa o ajustamento de duas ordens de fatores: de um lado, o comando genético determinando o que, potencialmente, deve acontecer; de outra parte, as condições biológicas e socioambientais, estabelecendo o que de fato se realiza como manifestação somática e funcional do crescimento, ou seja, o fenótipo. Como o programa genético praticamente não muda, os diferenciais de estatura ao longo de observações temporalmente referenciadas e entre espaços geográficos acham-se correlacionados a fatores de proteção ou de risco, favorecendo ou, ao contário, dificultando ou até impedindo o processo previsto pelas leis da herediariedade 1 .

Essa perspectiva conceitual possibilita ajuizar que a estatura de indivíduos, representando populações de diferentes gerações e espaços geográficos, expressa as condições de vida que contextualizaram seu ambiente de crescimento físico, desde a vida intrauterina ao final da adolescência ${ }^{2}$. Assim, a antropometria histórica, correlacionando a estatura de pessoas e populações em estudos de tendências temporais, presta-se muito bem para o próprio resgate dos ecossistemas de vida sob os quais se desenvolveu o processo somatométrico. Isso inclui, em primeira instância, a situação alimentar (macro e micronutrientes), as condições de saúde 
(saneamento, acesso e resolutividade das ações de saúde), nível de renda, situação educacional, estilos de vida e outros fatores correlatos. Assim, a antropometria torna-se um indicador holístico, de modo que o curso temporal, demográfico e espacial da estatura, mais do que um marcador epidemiológico, extrapola o campo da saúde propriamente dita para compreender o desenvolvimento humano em suas diferentes dimensões 3,4,5. É uma interpretação que, inclusive, passa a integrar novos conceitos e práticas de observações e análise das condições de vida, seja da parte de economistas tais como Sen 6, Fogel 7 e Sachs 8, ou de instituições como o Banco Mundial 9 e estudiosos sociais sobre alimentação e nutrição da Universidade de Paris 8 10,11. A tendência recente e universal do aumento de estatura é um evento marcante do século XX, não tendo sido uniforme no século XIX, como nos Estados Unidos e Inglaterra, onde foram bem documentados períodos de acentuada redução da estatura de adultos 12. Fênomeno semelhante foi observado no Brasil (Rio Grande do Sul), no período 1889-1920, como relatam Monastério \& Signorini 13, em decorrência das implicações históricas do desenvolvimento econômico e social daquele estado e do próprio país.

Atualmente, segundo a Organização das Nações Unidas, $34 \%$ das crianças de todo o mundo apresentam retardo de altura, e entre os países menos desenvolvidos tal percentual chega a $44 \% 14$. No caso brasileiro, é pertinente observar que poucos países em desenvolvimento apresentaram, nos últimos 60 anos, mudanças conjunturais tão notáveis e até conflitivas. Passamos de uma sociedade agropostoril tradicional para uma sociedade urbano-industrial de capitalismo periférico. Delineia-se, nesse processo, um invejável progresso material ao lado de um cenário social desfavorável: assim o Brasil ocupa uma posição privilegiada entre as sete maiores economias do mundo, enquanto se coloca entre os oito países mais desiguais em termos de distribuição de renda 15 . Simultaneamente, apresenta grandes disparidades inter-regionais, mudanças demográficas expressivas, coexistência de modelos antagônicos de morbimortalidade, declínio acentuado da natalidade e da mortalidade, caracterizando um intenso e rápido processo de transição epidemiológica e nutricional 16. Tratase, por conseguinte, de uma situação muito interessante para se estudar mudanças ocorridas na estatura de diferentes gerações.

Nessa perspectiva, objetiva-se, com o estudo aqui relatado, descrever o crescimento estatural de crianças, adolescentes e adultos no Estado de Pernambuco, no período mais marcante das grandes mudanças operadas no processo eco- nômico e social do Brasil e da Região Nordeste, ou seja, a partir da década de 1940. O Estado de Pernambuco é a unidade federativa melhor estudada do país, sob o ponto de vista de saúde e nutrição, com três inquéritos realizados no espaço de 15 anos. Este artigo constitui a etapa descritiva de uma avaliação que será complementada em outra publicação, fundamentalmente analítica, utilizando a mesma amostra.

\section{Métodos}

O estudo é do tipo observacional, analítico, quantitativo, temporalmente caracterizado como tendência secular. Os dados analisados provêm de duas pesquisas estaduais, realizadas simultaneamente entre maio e outubro de 2006: a III Pesquisa Estadual de Saúde e Nutrição: Saúde, Nutrição, Alimentação, Condições Socioeconômicas e Atenção à Saúde no Estado de Pernambuco (III PESN-PE/2006) e o I Inquérito Estadual sobre Doenças Crônicas e Agravos não Transmissíveis: Prevalência e Fatores de Risco (I DANT-PE/2006). Em relação a III PESN, o objetivo principal foi a atualização e a ampliação do diagnóstico da situação alimentar, nutricional, de saúde e condições socioeconômicas do Estado de Pernambuco. O estudo sobre as DANT foi um inquérito pioneiro e representativo da população, com 25 anos e mais do Estado de Pernambuco. Essa investigação teve como objetivos avaliar a prevalência e possíveis fatores de risco para doenças e agravos não transmissíveis, bem como analisar as condições estruturais e funcionais dos serviços públicos de saúde em relação à prevenção e ao tratamento dessas doenças.

O procedimento amostral e de coleta dos dados foi simultâneo nas duas pesquisas. Para a III PESN, o tamanho amostral foi definido para estimar dois parâmetros populacionais: as prevalências de desnutrição energético-proteica (DEP), segundo o critério peso/idade, em crianças menores de cinco anos, residentes nos estratos urbano e rural do Estado de Pernambuco. De acordo com as informações fornecidas pela II PESN, os valores esperados dessas prevalências eram, respectivamente, $3,2 \%$ e $6,2 \%$. Com um nível de 95\% de confiança, amostras de 762 crianças da zona urbana e 730 da zona rural seriam suficientes para estimar as correspondentes prevalências de DEP, com erro de estimação não superior a $1,25 \%$ e $1,75 \%$, respectivamente. Para compensar possíveis perdas e permitir desagregações com propósitos analíticos, os tamanhos amostrais foram aumentados em $10 \%$, passando para 839 e 803 , respectivamente, perfazendo um total de 1.642 crianças. Ainda como parte da 
III PESN, figuravam como objetivos estudar a situação nutricional de mães de crianças menores de cinco anos, bem como de mulheres em idade reprodutiva, excetuando-se as gestantes, por sua condição específica e baixa representação numérica (menos de $2 \%$ da população). Para esse segmento populacional, foram consideradas relevantes, para fins de cálculo amostral, prevalências reais ou presumíveis de $25 \%$ para anemia, $15 \%$ (presumível) para deficiência de vitamina A (DVA) e $40 \%$ para sobrepeso/obesidade. Para uma confiança de 95\% e erros de estimação de $3 \%$ para anemia e DVA e de $5 \%$ para sobrepeso/ obesidade, foram calculadas, respectivamente, amostras de tamanhos 800, 544 e 369.

Em relação à pesquisa DANT, o tamanho amostral foi definido com o objetivo de estimar sete parâmetros populacionais de interesse para o projeto: prevalências de sobrepeso/obesidade, hipertensão arterial sistêmica (HAS), hipercolesterolemia, hipertrigliceridemia, diabetes, tabagismo e sedentarismo, cujos valores esperados eram, respectivamente, $40 \%, 15 \%, 20 \%, 20 \%, 6 \%$, $20 \%$ e $40 \%$. Para um nível de confiança de $95 \%$ e correspondentes erros de estimação de $3 \%, 2 \%$, $2,5 \%, 1,5 \%, 2,5 \%$ e $3 \%$, o tamanho amostral máximo foi igual a 1.224. Com o propósito de compensar possíveis perdas e realizar desagregações com fins analíticos, esse tamanho amostral foi aumentado em 50\%, totalizando uma amostra planejada de 1.836 adultos.

Finalmente, por uma decisão da coordenação das duas pesquisas, considerou-se de interesse fazer uma avaliação antropométrica de toda família, incluindo-se na avaliação os escolares, adolescentes e adultos de 19 a 24 anos. Para esse novo conjunto populacional, admitindo-se um déficit de $12 \%$ para a relação A/I no final da adolescência, um erro amostral de $2 \%$ e um nível de 95\% de confiança, chegou-se ao cálculo de 1.013 pessoas, que foi aumentado em $30 \%$, passando para 1.317, a fim de compensar perdas e permitir estratificações com fins analíticos. Como consequência de tais decisões, a amostra total para as duas pesquisas foi de 6.508 pessoas $(1.642+$ $800+544+369+1.836+1.317)$. A amostra final ficou em 6.471 sujeitos.

Foram selecionadas amostras probabilísticas representativas dos três estratos em que foi dividido o espaço geográfico estadual: Região Metropolitana do Recife, Interior Urbano e Interior Rural. O procedimento amostral foi executado em três estágios. No primeiro e segundo foram selecionados os municípios de cada estrato geográfico e os setores censitários de cada município. No terceiro estágio, sorteou-se em cada setor censitário um ponto inicial a partir do qual, em sentido anti-horário, foram localizadas as unidades domiciliares onde residiam crianças menores de cinco anos ou adultos com mais de 25 anos. Ao final do processo amostral, haviam sido sorteados 1.314 domicílios, com o total de 6.471 moradores, que foram os sujeitos das duas pesquisas.

O trabalho de campo foi realizado por uma equipe previamente treinada com a finalidade de padronizar as ações de coleta dos dados, que foram obtidos com aplicação de questionários com perguntas pré-codificadas referentes às informações socioeconômicas, demográficas e biológicas. Os dados antropométricos foram registrados em formulário específico depois de coletados por antropometristas treinados, adotando-se os procedimentos técnicos recomendados pela Organização Mundial da Saúde (OMS) 17 e as normas do Manual de Acompanhamento do Crescimento e Desenvolvimento Infantil do Ministério da Saúde 18.

A aferição da estatura, a partir de 24 meses, foi realizada com estadiômetro portátil (Alturexata Ltda., Belo Horizonte, Brasil), de precisão igual $1 \mathrm{~mm}$. Nas crianças abaixo de 24 meses, o comprimento foi registrado com a criança em decúbito dorsal, utilizando-se um infantômetro (Rollameter by Razen, Raven Equipment, Great Dunmow, Inglaterra), com precisão de $1 \mathrm{~mm}$. Para garantir a precisão e exatidão das mensurações, dois técnicos treinados realizavam individualmente e de forma independente uma aferição da estatura de cada sujeito. Quando a diferença entre essas duas medidas não excedia $0,5 \mathrm{~cm}$, sua média era registrada como a estatura do sujeito. Quando o limite de $0,5 \mathrm{~cm}$ era ultrapassado, realizava-se uma terceira medida e registrava-se a média das duas mais próximas.

A altura foi sistematicamente registrada entre as pessoas que nasceram a partir de 1945 , perfazendo um total de 5.319, com a seguinte distribuição etária: 1.611 menores de cinco anos, 1.391 crianças e adolescentes (5 a 19 anos) e 2.317 adultos. Foi esse conjunto amostral que possibilitou a elaboração do presente estudo, descrevendo, numa perspectiva diacrônica, o comportamento intergeracional do crescimento estatural no Estado de Pernambuco, desde a metade dos anos 40 até 2006.

\section{Análise estatística}

Os dados numéricos foram resumidos por médias e desvio-padrão; os dados categóricos, por frequências absolutas e percentuais. Para descrever o crescimento estatural, foram ajustados modelos de regressão linear com interceptos aleatórios, devido à natureza hierárquica do arranjo populacional (moradores aninhados em 
domicílios), respeitada no procedimento amostral. A adoção de modelos de regressão linear foi sugerida pelos resultados de uma análise exploratória prévia utilizando spline cúbica 19.

Para os adultos, a variável resposta foi a estatura; para crianças e adolescentes, o escore-z do índice altura/idade (ZAI), seguindo-se o procedimento usual de adotar essa escala para expressar o desenvolvimento estatural desses grupos etários. Os escores ZAI de crianças e adolescentes foram obtidos com o emprego do programa Anthro Plus, versão 1.0.2 (Organização Mundial da Saúde; http://www.who.int/growthref/tools / en/). Três sujeitos com valores de ZAI menores que -5 e outros 10 , com valores maiores que +3 , foram excluídos da análise por serem considerados implausíveis ou excepcionais segundo recomendação de Grummer-Strawn 20. Em cada modelo, a variável exploratória foi o ano de nascimento, entendida como a data de nascimento registrada na forma decimal, após a conversão do dia e mês de nascimento em fração do ano (por exemplo: ano de nascimento igual a 2003.5 significa o nascimento ocorrido em 30/ Jun/2003). A data de nascimento expressa dessa forma funciona como uma proxy da idade cronológica, tornando mais conveniente a visualização gráfica da tendência temporal do crescimento da estatura, visto que ambas tendem a mostrar uma correlação positiva.

Com a utilização dos modelos ajustados, foram obtidas para cada idade e sexo uma estimativa pontual e uma estimativa intervalar (com nível de 95\% de confiança) para a correspondente estatura média. A estatura preconizada pela OMS (2006) ${ }^{21}$ para os jovens de 19 anos foi adotada como referencial para a estatura dos adultos do estudo. Nos testes de hipóteses, adotou-se o nível de significância de 0,05. A análise estatística dos dados foi realizada com os softwares R 2.12.3 (The R Foundation for Statistical Computing, Viena, Áustria; http: / / www.r-project.org ) e Stata SE 10.1 (Stata Corp., College Station, Estados Unidos).

\section{Aspectos éticos}

Os projetos de pesquisa da III PESN-PE/2006 e da I DANT-PE/2006 foram aprovados, em novembro de 2005 e janeiro de 2006, respectivamente, pelo Comitê de Ética em Pesquisa do Instituto de Medicina Integral Professor Fernando Figueira (IMIP).

\section{Resultados}

A Tabela 1 apresenta a descrição dos sujeitos do estudo, com relação ao sexo, idade, peso, altura, residência e escolaridade. A Figura 1 evidencia a presença de uma tendência secular bem definida de crescimento estatural em função da data de nascimento, pois os coeficientes de regressão dos modelos ajustados foram todos significantes (teste Z: p < 0,001 em cada modelo). Nos adultos, as taxas de crescimento dos homens e das mulheres foram diferentes, já que a interação entre ano de nascimento e sexo foi significante $(p=0,002)$. A estatura média dos homens

\section{Tabela 1}

Algumas características amostrais de crianças, adolescentes e adultos nascidos entre 1945 e 2006 e residindo em Pernambuco, Brasil, no momento da realização das pesquisas DANT e III PESN (maio a outubro de 2006).

\begin{tabular}{|c|c|c|c|}
\hline Características & $\begin{array}{l}\text { Crianças } \\
\text { (<5 anos) } \\
{[n=1.611]}\end{array}$ & $\begin{array}{l}\text { Crianças e adolescentes } \\
\qquad \begin{array}{c}\text { (5-19 anos) } \\
{[n=1.391]}\end{array}\end{array}$ & $\begin{array}{c}\text { Adultos } \\
{[n=2.317]}\end{array}$ \\
\hline Sexo masculino [n (\%)] & $825(51,2)$ & $621(44,6)$ & $820(35,4)$ \\
\hline Idade (anos) [média \pm DP] & $2,5 \pm 1,4$ & $10,6 \pm 4,0$ & $32,8 \pm 9,9$ \\
\hline Peso (kg) [média $\pm \mathrm{DP}]$ & $12,8 \pm 3,9$ & $33,6 \pm 14,8$ & $64,8 \pm 13,8$ \\
\hline Altura $(\mathrm{cm})[$ média $\pm \mathrm{DP}]$ & $87,2 \pm 14,3$ & $135,6 \pm 19,1$ & $160,5 \pm 8,6$ \\
\hline Residência: área urbana [n (\%)] & $825(51,2)$ & $553(39,8)$ & $1.220(52,6)$ \\
\hline Escolaridade (anos) [média \pm DP] & - & $3,2 \pm 2,6$ * & $5,4 \pm 3,7$ ** \\
\hline
\end{tabular}

DANT: I Inquérito Estadual sobre Doenças Crônicas e Agravos não Transmissíveis: Prevalência e Fatores de Risco; DP: desvio-padrão; III PESN: III Pesquisa Estadual de Saúde e Nutrição: Saúde, Nutrição, Alimentação, Condições Socioeconômicas e Atenção à Saúde no Estado de Pernambuco.

* Informação disponível só em 1.080 sujeitos;

** Informação disponível só em 2.297 sujeitos. 
Crescimento estatural de crianças, adolescentes e adultos nascidos entre 1945-2006 e residindo em Pernambuco, Brasil, no momento da realização das pesquisas DANT e III PESN, realizadas de maio a outubro de 2006.

1a) Adultos masculinos

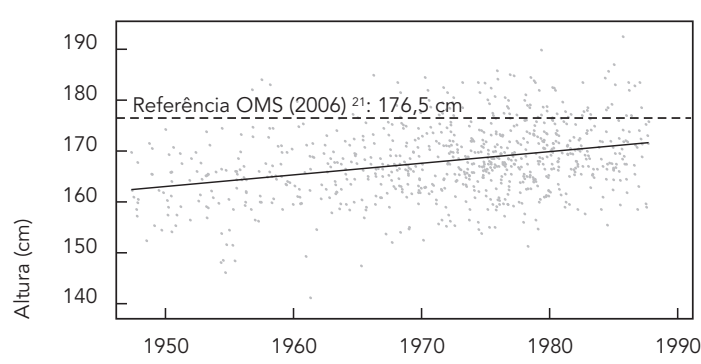

1c) Crianças e adolescentes masculinos

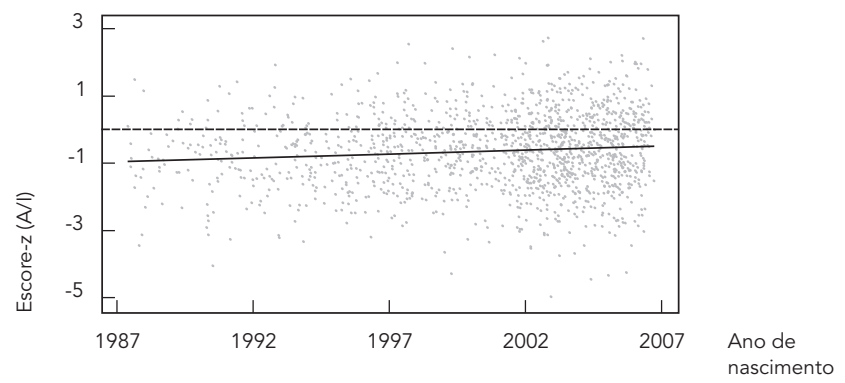

1b) Adultos femininos

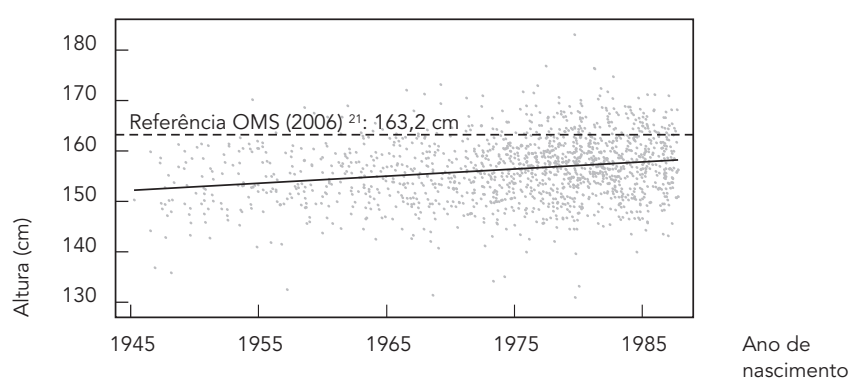

1d) Crianças e adolescentes femininos

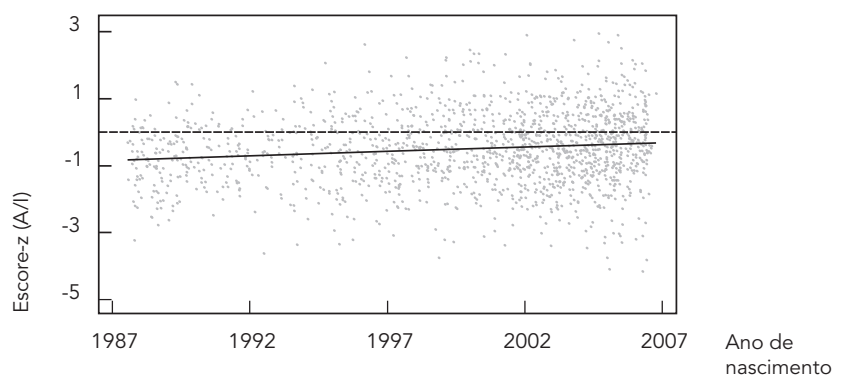

DANT: I Inquérito Estadual sobre Doenças Crônicas e Agravos não Transmissíveis: Prevalência e Fatores de Risco; III PESN: III Pesquisa Estadual de Saúde e Nutrição: Saúde, Nutrição, Alimentação, Condições Socioeconômicas e Atenção à Saúde no Estado de Pernambuco.

elevou-sede 162,1cm em 1947 para 171,5 em 1987, crescendo a uma taxa aproximada de 0,23cm por ano (IC95\%: 0,19-0,28). Entre as mulheres, a estatura média aumentou de $151,7 \mathrm{~cm}$ em 1945 para $158,2 \mathrm{~cm}$ em 1987, com uma taxa de crescimento estimada em $0,15 \mathrm{~cm}$ por ano (IC95\%: 0,13-0,18). Embora crescimento estatural de homens e mulheres adultos tenha se mantido crescente entre 1945 e 1987, verifica-se, pelo exame dos intervalos de confiança da Tabela 2 , que as estaturas médias desses dois grupos sempre ficaram significativamente abaixo do padrão estabelecido pela OMS. A comparação com esse padrão revela que, entre os homens, o déficit estatural decresceu de 14,4cm, em 1947, para 5,0cm, em 1987, apresentando, assim, uma diminuição relativa de 65,3\%. Entre as mulheres, o déficit baixou de $11,5 \mathrm{~cm}$, em 1945, para 5,0cm em 1987, com uma redução proporcional de $56,5 \%$.
No segmento ainda em fase de crescimento estatural durante a realização dos inquéritos (menores de 19 anos), não houve interação estatisticamente significante entre o sexo e ano de nascimento (teste $\mathrm{Z}: \mathrm{p}=0,678$ ). Em relação aos padrões da OMS, as crianças e os adolescentes apresentaram déficit estatural declinante no período de 1987-2006. Nos dois grupos, o exame dos intervalos de confiança das médias (Tabela 3) mostra uma defasagem sistemática em relação à referência da OMS, mas o déficit diminuiu de forma continuada no sentido das gerações mais novas, em ambos os sexos. De fato, o déficit estatural declinou, no período de 1987-2006, a taxa de -0,013 escores-z do indicador A/I, para o sexo masculino e -0,019 para sexo feminino. Por outro lado, como ilustra a Figura 2, a estatura média sempre esteve acima de - 1 escore- $z$ do indicador A/I sendo, em geral, mais próxima desses valores no sexo masculino do que no sexo feminino. Por 
Estimativas pontual * e intervalar das estaturas médias de homens e de mulheres residentes em Pernambuco, Brasil, no ano de 2006, de acordo com a data de nascimento.

\begin{tabular}{|c|c|c|c|c|}
\hline \multirow[t]{2}{*}{ Ano de nascimento } & \multicolumn{4}{|c|}{ Estatura média estimada $(\mathrm{cm})$} \\
\hline & Masculino ** & IC95\% & Feminino $* \star \star$ & IC95\% \\
\hline 1945 & - & - & 151,7 & $150,8-152,6$ \\
\hline 1947 & 162,1 & $161,0-163,3$ & 152,0 & $151,1-152,9$ \\
\hline 1950 & 162,8 & $161,8-163,9$ & 153,0 & $152,5-153,2$ \\
\hline 1955 & 164,0 & $163,1-164,9$ & 153,2 & $152,6-153,9$ \\
\hline 1960 & 165,2 & $164,5-165,9$ & 154,0 & $153,5-154,5$ \\
\hline 1965 & 166,3 & $165,8-166,9$ & 154,8 & $154,3-155,2$ \\
\hline 1970 & 167,5 & $167,0-168,0$ & 155,5 & $155,2-155,9$ \\
\hline 1975 & 168,7 & $168,2-169,2$ & 156,3 & $156,0-156,6$ \\
\hline 1980 & 169,9 & $169,3-170,5$ & 157,1 & $156,7-157,4$ \\
\hline 1985 & 171,0 & $170,3-171,8$ & 157,9 & $157,4-158,3$ \\
\hline
\end{tabular}

IC95\%: intervalo de 95\% de confiança.

* Modelo ajustado (masculino): estatura média estimada $=162,1+0,23 \times$ ano de nascimento; Modelo ajustado (feminino):

estatura média estimada $=151,7+0,15 \times$ ano de nascimento;

** Padrão OMS (2006) 21: 176,5cm;

*** Padrão OMS (2006) 21: 163,2cm.

outro lado, os homens e as mulheres que completaram 19 anos em 1987, apresentaram déficits de estatura de 5,0cm. Projetando-se prospectivamente as tendências verificadas, as populações adultas masculina e feminina do Estado de Pernambuco esperariam, respectivamente, em torno de 22 e 33 anos para atingir o padrão internacional preconizado pela OMS. Por outro lado, tendo-se em conta que o déficit estatural de menores de cinco anos decresceu de $12,1 \%$ em 1997 (II PESN) para 6,4\% em 2006 (III PESN), o que corresponde a um decréscimo anual de $0,63 \%$, estima-se que, mantendo-se tal decréscimo, dentro de 6 a 7 anos, a proporção do déficit estatural dessas crianças chegaria a 2,3\%, que é a ocorrência verificada no padrão internacional de normalidade antopométrica da OMS, compensando, assim, o histórico retardo de crescimento somatométrico da população mais jovem de Pernambuco.

\section{Discussão}

A comparação dos resultados aqui expostos, seja no plano interno (sequências de observações referentes à estatura da população em crescimento: crianças, adolescentes e adultos), seja no plano externo, pressupondo como referência os valores padrão recomendados pela OMS, demonstram a magnitude e a rapidez das mudan- ças que estão ocorrendo no estado nutricional da população do Estado de Pernambuco, nas últimas seis décadas.

Em relação às análises intergrupais da amostra, evidenciam-se claramente as notáveis diferenças entre a coorte dos nascidos entre 1945 e 1987, representando o segmento de adultos e a evolução antropométrica das crianças e adolescentes como representação do segmento biológico em fase de crescimento estatural. Nesse sentido, convém assinalar que, de 1950 até 1987 , entre os homens adultos, observou-se um aumento cumulativo de $8,7 \mathrm{~cm}$, ou seja, $0,22 \mathrm{~cm}$ por ano. Para as mulheres adultas, entre os extremos da série temporal (1945-1987), o aumento verificado foi de $5,5 \mathrm{~cm}$, o que equivale, como média anualizada, a um incremento de $0,14 \mathrm{~cm}$ por ano, bem inferior, portanto, ao que ocorreu entre os homens. A discussão dessas diferenças de valores absolutos e relativos será retomada na análise dos resutados referentes às comparações "externas”, vale dizer, em relação aos padrões intenacionais da OMS.

Já nos nascidos a partir de 1987, constituindo o conjunto amostral de crianças e adolescentes, o comportamento foi bem diverso: para um período de 19 anos, os ganhos alcançados em termos médios de evolução estatural foram proporcionalmente maiores que os obtidos pelos adultos (geração de pais a avós) no curso de quase 50 anos. (Os termos pais e avós aqui empregados 


\section{Figura 2}

Evolução da estatura média de crianças e adolescentes nascidos entre 1987-2006 e residentes em Pernambuco, Brasil, no momento da realização das pesquisas DANT e III PESN, realizadas de maio a outubro de 2006.

2a) Masculino

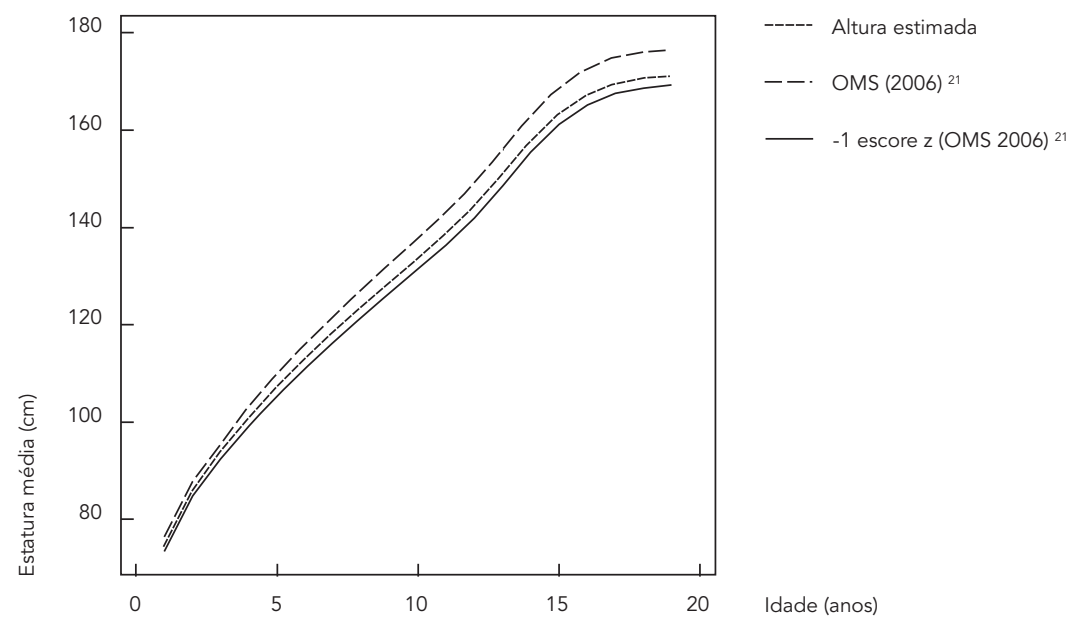

2b) Feminino

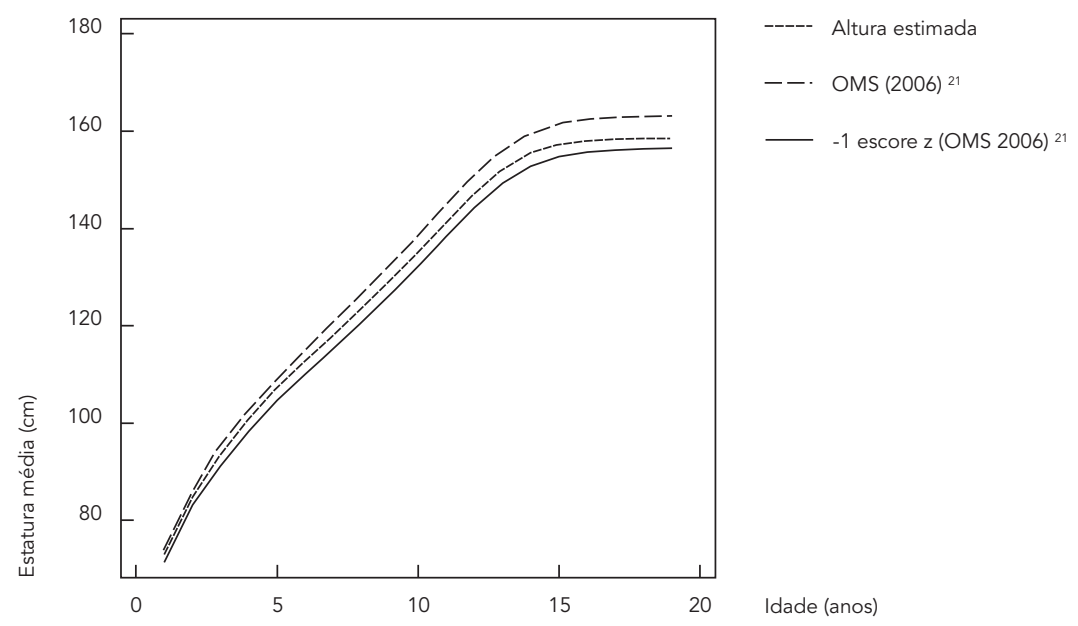

DANT: I Inquérito Estadual sobre Doenças Crônicas e Agravos não Transmissíveis: Prevalência e Fatores de Risco; III PESN: III Pesquisa Estadual de Saúde e Nutrição: Saúde, Nutrição, Alimentação, Condições Socioeconômicas e Atenção à Saúde no Estado de Pernambuco. 
Estimativa pontual do escore ZAI (escore-z do índice altura/idade) *, estimativas pontual e intervalar da estatura média, referência OMS (2006) 21 para estatura. Crianças e adolescentes de ambos os sexos, nascidos entre 1987-2006 e residentes em Pernambuco, Brasil, no último ano desse período.

\begin{tabular}{|c|c|c|c|c|}
\hline Sexo/Idade (anos) & ZAl estimado & $\begin{array}{l}\text { Estatura média } \\
\text { estimada }(\mathrm{cm})\end{array}$ & IC95\% & OMS (Referência) \\
\hline \multicolumn{5}{|l|}{ Masculino } \\
\hline 1 & $-0,52$ & 74,5 & $74,3-74,7$ & 75,7 \\
\hline 2 & $-0,53$ & 86,2 & $86,0-86,4$ & 87,8 \\
\hline 3 & $-0,54$ & 94,1 & $93,8-94,3$ & 96,1 \\
\hline 4 & $-0,56$ & 101,0 & $100,7-101,3$ & 103,3 \\
\hline 5 & $-0,57$ & 107,3 & $107,0-107,6$ & 110,0 \\
\hline 6 & $-0,58$ & 113,1 & $112,8-113,4$ & 116,0 \\
\hline 7 & $-0,59$ & 118,6 & $118,2-119,0$ & 121,7 \\
\hline 8 & $-0,61$ & 123,8 & $123,4-124,3$ & 127,3 \\
\hline 9 & $-0,62$ & 128,8 & $128,4-129,3$ & 132,6 \\
\hline 10 & $-0,63$ & 133,7 & $133,2-134,3$ & 137,8 \\
\hline 11 & $-0,64$ & 138,8 & $138,1-139,4$ & 143,1 \\
\hline 12 & $-0,66$ & 144,4 & $143,7-145,1$ & 149,1 \\
\hline 13 & $-0,67$ & 151,1 & $150,2-151,9$ & 156,0 \\
\hline 14 & $-0,68$ & 157,9 & $157,0-158,8$ & 163,2 \\
\hline 15 & $-0,70$ & 163,5 & $162,5-164,5$ & 169,0 \\
\hline 16 & $-0,71$ & 167,4 & $166,3-168,5$ & 172,9 \\
\hline 17 & $-0,72$ & 169,7 & $168,5-170,8$ & 175,2 \\
\hline 18 & $-0,73$ & 170,7 & $169,5-171,9$ & 176,1 \\
\hline 19 & $-0,75$ & 171,1 & $169,9-172,3$ & 176,5 \\
\hline \multicolumn{5}{|l|}{ Feminino } \\
\hline 1 & $-0,36$ & 73,1 & $72,9-73,3$ & 74,0 \\
\hline 2 & $-0,37$ & 85,2 & $85,0-85,4$ & 86,4 \\
\hline 3 & $-0,39$ & 93,6 & $93,3-93,8$ & 95,1 \\
\hline 4 & $-0,41$ & 100,9 & $100,7-101,2$ & 102,7 \\
\hline 5 & $-0,43$ & 107,4 & $107,1-107,7$ & 109,4 \\
\hline 6 & $-0,45$ & 112,8 & $112,5-113,1$ & 115,1 \\
\hline 7 & $-0,47$ & 118,2 & $117,9-118,6$ & 120,8 \\
\hline 8 & $-0,49$ & 123,7 & $123,3-124,1$ & 126,6 \\
\hline 9 & $-0,51$ & 129,4 & $129,0-129,8$ & 132,5 \\
\hline 10 & $-0,53$ & 135,2 & $134,8-135,7$ & 138,6 \\
\hline 11 & $-0,55$ & 141,3 & $140,8-141,8$ & 145,0 \\
\hline 12 & $-0,57$ & 147,3 & $146,8-147,9$ & 151,2 \\
\hline 13 & $-0,59$ & 152,3 & $151,7-152,9$ & 156,4 \\
\hline 14 & $-0,61$ & 155,6 & $154,9-156,2$ & 159,8 \\
\hline 15 & $-0,63$ & 157,4 & $156,7-158,0$ & 161,7 \\
\hline 16 & $-0,65$ & 158,1 & $157,4-158,9$ & 162,5 \\
\hline 17 & $-0,67$ & 158,4 & $157,6-159,2$ & 162,9 \\
\hline 18 & $-0,69$ & 158,5 & $157,7-159,3$ & 163,1 \\
\hline 19 & $-0,70$ & 158,5 & $157,7-159,4$ & 163,2 \\
\hline
\end{tabular}

IC95\%: intervalo de 95\% de confiança; OMS: Organização Mundial da Saúde.

* Modelo ajustado (masculino): escore ZAl médio estimado $=-0,80+0,031 \times$ ano de nascimento;

Modelo ajustado (feminino): escore ZAl médio estimado $=-0,90+0,019 \times$ ano de nascimento. 
significam uma designação em parte simbólica aplicada a adultos que, necessarimente, não são pais ou avós ou poderiam ser até mesmos bisavós). É bem ilustrativa a demonstração de que aos 19 anos, adolescentes dos dois sexos já apresentam médias estaturais bem acima dos valores da coorte de adultos, embora ainda abaixo dos valores centrais do padrão da OMS. Por outro lado, se forem consideradas as médias de crianças com um ano de idade, percebe-se que se afastam apenas $0,9 \mathrm{~cm}$ (meninos) e $1,3 \mathrm{~cm}$ (meninas) das médias internacionais de normalidade antropométrica. Ademais, ao serem considerados especificamente os resultados das crianças menores de cinco anos, expressos em escores- $z$ da relação altura/idade, encontra-se que os afastamentos negativos se aproximam rapidamente dos valores médios esperados de uma população normal.

Delineam-se, portanto, três grandes diferenças na avaliação estatural da população do Estado de Pernambuco analisada na perspectiva de tendência secular: as variações bem significativas da coorte que, teoricamente, já completou seu ciclo de crescimento, caracteriza-se por um ritmo lento de mudanças, contrapondo-se à população de crianças e adolescentes, quando referenciadas com a linha do padrão internacional, em valores absolutos e posições relativas (escores-z). De fato, se na evolução temporal da coorte de crianças e adolescentes se observar a relação idade/estatura, infere-se que, quanto mais jovem, mais a criança se aproxima da linha referencial de normalidade. Configura-se, neste comportamento estatístico, a nítida ocorrência da transição nutricional no Brasil, caracterizada pela rápida redução dos déficits antropométricos. Essa observação, no caso de Pernambuco, corrobora com a verificação de que foi (ou é) no Nordeste que, em anos recentes, mais se acentuou o declínio da desnutrição em crianças 22.

É pertinente referir que, objetivando estudar a tendência secular sobre a evolução estatural de crianças e adultos, Monteiro et al. 23 analisaram diferenças de estatura com 21 anos de idade $( \pm 12$ meses) e de escolares de sete anos ( \pm 12 meses) em dois inquéritos nutricionais de abrangência nacional: o Estudo Nacional de Despesas Familiares (ENDEF) e a Pesquisa Nacional de Saúde e Nutrição (PNSN), ambos realizados pelo Instituto Brasileiro de Geografia e Estatística (IBGE), o primeiro entre 1974/1975 e o segundo, em 1989, com uma diferença de 14 anos entre os dois inquéritos. Na estratégia de análise foram representados três grupos: (a) adultos nascidos entre 1951/1953; (b) adultos nascidos entre 1966/1968; (c) crianças nascidas no biênio 1982/1983. Ainda que de nível nacional e aplicado a faixas limitadas de representação etária (sete anos e 21/22 anos), além de cobrir um espaço temporal compreendido entre 1951 e 1983 (portanto, com três diferenças nos elementos básicos de comparação), esse estudo é de marcante pertinência para os objetivos considerados no presente artigo, envolvendo gerações nascidas entre 1945 e 2006, no Estado de Pernambuco.

Apesar das discrepâncias de tempo, espaço e amostra, o estudo de Monteiro et al. 23 oferece suporte bem consistente para a discussão dos resultados aqui expostos. Nesse sentido, é interessante destacar que entre adultos jovens (2122 anos), o aumento de estatura registrado no Brasil foi de $1,3 \mathrm{~cm}$ para homens e $1,0 \mathrm{~cm}$ para as mulheres. Já entre as crianças de 7 anos, o efeito diferencial entre os dois grupos comparados foi bem mais acentuado: $3,6 \mathrm{~cm}$ para os meninos e $3,7 \mathrm{~cm}$ para as meninas. Entre os escolares, a redução do déficit de estatura foi três vezes maior, alcançando aproximadamente $50 \%$, registrando um declínio de $7,3 \mathrm{~cm}$ para $3,7 \mathrm{~cm}$ nos meninos e de $6,6 \mathrm{~cm}$ para $2,9 \mathrm{~cm}$ entre as meninas.

Fica assim bem evidenciado que no Brasil e na Região Nordeste, a evolução temporal da estatura média se comportou de forma bem diferenciada entre indivíduos em fase de crescimento e aqueles que já alcançaram a idade adulta. $\mathrm{O}$ mesmo ocorreu no Estado de Pernambuco, relativizando-se, como já foi notificado, os efeitos representados pela amostragem (séries contínuas em termos de idade para ingresso de crianças e adultos no caso de Pernambuco e coortes com idade e datas seletivas de nascimento pré-fixadas no caso do inquérito nacional). Ademais, usando padrões referenciais diferentes (NCHS 1977 24, para o estudo nacional, OMS $2006{ }^{21}$ para o Estado de Pernambuco), estabelecem-se diferenças metodológicas que relativizam as comparações. No entanto, os vieses resultantes são de pequeno porte, de forma que o estudo de Monteiro et al. 22 ainda representa a situação mais apropriada para discutir os resultados aqui analisados.

O que acontece com a população brasileira e, de forma mais explícita, no caso de Pernambuco, caracteriza um processo novo e surpreendente, como conceito, consequências e desafios para a agenda de saúde coletiva dos tempos recentes (pós-1950) e suas projeções para o futuro: a transição epidemiológica 25,26 e, sobretudo, seu componente nutricional 16,27. De fato, é muito ilustrativo que, no breve espaço de 60 anos, transformações na estrutura epidemiológica da saúde e nutrição tenham mudado tão substancialmente a situação nutricional em nível de população. A propósito, cumpre ressaltar que as transformações no perfil de morbimortalidade registrada no mundo, notadamente nos países mais desenvolvidos 28 , decorreram em ritmo 
mais lento do que está acontecendo na América Latina 29 e, muito particularmente, no Brasil 30. Monteiro et al. 22 ressaltam que no caso brasileiro é um tanto singular no contexto internacional, exatamente pela rápida diminuição na prevalência da DEP e emergência acelerada do complexo de morbidades que constituem as doenças crônicas não transmissíveis, tendo a díade sobrepeso/obesidade como representação mais típica 22. Por outro lado, se o caso brasileiro pode ser considerado como um processo surpreendente pela rapidez das mudanças produzidas, o caso de Pernambuco, aqui representado pela progressiva e rápida sucessão de mudanças do déficit estatural, pode ser considerado também como mais surpreendente dentro do próprio contexto nacional, por se tratar, juntamente com o Norte, da região mais pobre do Brasil. Nesse sentido, caberia especular que a situação de Pernambuco provavelmente expressa o que está ocorrendo no Nordeste como um todo. E, na medida em que o processo de transição nutricional que se intensificou e se particularizou como um evento de características um tanto singulares no Brasil, a experiência do Estado de Pernambuco, aqui relatada, representando o seguimento de diferentes gerações, grande parte das quais encadeadas por laços familiares, torna-se um objeto de interesse acadêmico que extrapola os limites de sua própria configuração geográfica e espacial do Estado.

É oportuno e pertinente considerar que na avaliação temporal de populações adultas os ganhos estaturais não expressam apenas os ganhos diacrônicos do período de crescimento; revelam ainda, um processo oposto, ou seja, as perdas decorrentes do envelhecimento, por di- minuição dos discos intervertebrais, posturas viciosas da coluna e novas angulações das articulações dos membros inferiores. Por conseguinte, na coorte de adultos e idosos, opera-se um balanço do que se ganha na infância e adolescência com o que progressivamente se perde, depois dos 40 anos. E, evidentemente, o saldo deve ser sistematicamente negativo aos 50, 60 e mais anos 31. Cabe informar que o trabalho apresenta evidentes limitações, duas das quais são destacadas: (a) não se tratou de uma pesquisa desenhada especificamente para os objetivos aqui explicitados, constituindo de fato a utilização de um banco de dados combinando dois projetos diferentes, mas complementares. Não reúne, portanto, todo o conjunto de informações que teoricamente seria desejável; (b) o segundo aspecto deriva da condição de que uma das pesquisas representa a continuidade, em perspectiva temporal, de um primeiro estudo realizado em 1991 implicando, em grande parte, a manutenção de objetivos e procedimentos pré-fixados há 20 anos. Isso impõem roteiros e rotinas preestabelecidos que, obviamente, já não seriam completamente adotados num projeto proposto para os dias atuais.

Em resumo, é importante concluir que, enquanto se estimaria que a correção do déficit de estatura da população do Estado de Pernambuco (27 anos para os homens e 36 anos para as mulheres), se mantidos os ganhos históricos dos adultos, a correção seria alcançada entre seis e sete anos, se for considerada a tendência estatística alcançada pelos menores de cinco anos. É uma perspectiva animadora, considerando Pernambuco como parte da região mais pobre do país: o Nordeste brasileiro. 


\section{Resumo}

Utilizando-se modelos de regressão linear com interceptos aleatórios, foi realizada uma descrição da evolução estatural de pessoas residentes em Pernambuco, Brasil, em 2006 e nascidas a partir de 1945. O ganho estatural dos homens adultos foi de $0,23 \mathrm{~cm} /$ ano, entre 1947-1987; das mulheres, 0,15cm/ano, entre 1945-1987. Em relação aos padrões da Organização Mundial da Saúde (OMS 2006), as crianças e os adolescentes apresentaram déficit estatural declinante no período de 1987-2006, com uma taxa anual de -0,019 escores- $z$ (A/I) para o sexo feminino e $-0,013$, para o sexo masculino; os homens e as mulheres que completaram 19 anos em 1987, apresentaram déficit de estatura de 5,0cm. Projetando-se as tendências observadas em 2006, os homens e as mulheres adultos de Pernambuco esperariam, respectivamente, em torno de 22-33 anos para atingir o padrão internacional. Por sua vez, as crianças menores de cinco anos apresentariam, dentro de 6-7 anos, um déficit estatural de 2,3\%, que é a ocorrência verificada no padrão da OMS, corrigindo, assim, o histórico retardo de crescimento dessa população em Pernambuco.

Antropometria; Crescimento e Desenvolvimento; Estatura-Idade

\section{Colaboradores}

J. N. Figueiroa participou de todas as etapas do trabalho: concepção, análise dos dados, discussão e elaboração do artigo. J. G. B. Alves contribuiu na elaboração, organização e revisão crítica do artigo. P. I. C. Lira contribuiu na análise dos dados, redação e revisão crítica do artigo. M. Batista Filho participou da conceçpão do trabalho, na redação, revisão crítica e aprovação final do artigo.

\section{Agradecimentos}

Os autores fazem um agradecimento especial à professora Tereza Cristina Miglioli pela inestimável ajuda prestada na finalização deste estudo.

\section{Referências}

1. Tanner JM. Foetus into man. Cambridge: Harvard University Press; 1990.

2. Steckel RH. Stature and the standard of living. J Econ Lit 1995; 33:1903-40.

3. Touraine A. Um novo paradigma - para compreender o mundo de hoje. Petrópolis: Editora Vozes; 2006.

4. Conway G. Produção de alimentos no século XXI: biotecnologia e meio ambiente. São Paulo: Estação Liberdade; 2003.

5. Abramovay R. Alimentos versus população: está ressurgindo o fantasma malthusiano? Ciênc Cult 2010; 62:38-42.

6. Sen A. Desenvolvimento como liberdade. São Paulo: Companhia das Letras; 2001.

7. Fogel RW. Health, nutrition, and economic growth. Econ Dev Cult Change 2004; 52:643-58.

8. Sachs I. Caminhos para o desenvolvimento sustentável. 3ạ Ed. Rio de Janeiro: Editora Garamond; 2008.

9. Musgrove P, editor. Health economics in development. Washington DC: World Bank; 2004.
10. Fishler C, Masson E. Manger, Français, Européens, et Americains face à l'alimentation. Paris: Odile Jacob; 2008.

11. Poulain JP. Sociologie de l'obesité. Paris: PUF; 2009.

12. Komlos J, Cinnirella F. European heights in the early 18th century. http://epub.ub.uni-muenchen. de/572/1/european_heights_in_the_early_18th_ century.pdf (acessado em 02/Mai/2011).

13. Monastério LM, Signorini MJS. As condições de vida dos gaúchos entre 1889-1920: uma análise antropométrica. Economia Selecta 2008; 9:111-26.

14. Fundo das Nações Unidas para a Infância. Situação mundial da infância. New York: Fundo das Nações Unidas para a Infância; 2011.

15. Instituto de Pesquisa Econômica Aplicada. Pobreza, desigualdade e políticas públicas. 2010. (Comunicados da Presidência, 38). http://www. asabrasil.org.br/UserFiles/File/Pobreza_desigual des_pp_ipea.pdf (acessado em 21/Dez/2011).

16. Popkin BM. Nutritional pattern and transition. Popul Dev Rev 1993; 19:138-57. 
17. World Health Organization. Physical status: the use and interpretation of anthropometry. Geneva: World Health Organization; 1995. (WHO Technical Report Series, 854).

18. Departamento de Atenção Básica, Secretaria de Políticas de Saúde, Ministério da Saúde. Saúde da criança: acompanhamento do crescimento e desenvolvimento infantil. Brasília: Ministério da Saúde; 2002. (Série Cadernos de Atenção Básica, 11. Série A. Normas e Manuais Técnicos).

19. Keele L. Semiparametric regression for the social sciences. West Sussex: John Wiley \& Sons; 2008.

20. Grummer-Strawn LM, Cáceres JM, Herrera de Jaimes BP. Trends in the nutritional status of Salvadorian children: the post-war experience. Bull World Health Organ 1966; 74:369-74.

21. WHO Multicentre Growth Reference Study Group. Enrolment and baseline characteristics in the WHO Multicentre Growth Reference Study. Acta Paediatr Suppl 2006; 450:7-15.

22. Monteiro CA, Benício MH, Konno SC, Silva ACF, Lima ALL, Conde WL. Causas do declínio da desnutrição infantil no Brasil, 1996-2007. Rev Saúde Pública 2009; 43:35-43.

23. Monteiro CA, Benício MH, Gouveia NC. Secular growth trends in Brazil over three decades. Ann Hum Biol 1994; 21:381-90.
24. Hamill PV, Drizd TA, Johnson CL, Reed CB, Roche AF. NCHS growth curves for children birth-18 years. United States. Vital Health Stat 11 1977; (165):1-74.

25. Frederiksen H, Feedbacks I. Economic and demographic transition. Science 1969; 166:837-47.

26. Omran AR. The epidemiology transition: a theory of the epidemiology of population change. Milbank Mem Fund Q 1971; 49:509-38.

27. Batista Filho M, Assis AM, Kac G. Transição nutricional: conceito e características. In: Kac G, Sichieri R, Gigante DP, organizadores. Epidemiologia nutricional. Rio de Janeiro: Editora Fiocruz/Editora Atheneu; 2007. p. 445-60.

28. Monteiro CA, Moura EC, Conde WL, Popkin BM. Socioeconomic status and obesity in adult population of developing countries: a review. Bull World Health Organ 2004; 82:940-6.

29. Kac G, Velásquez-Meléndez G. A transição nutricional e a epidemiologia da obesidade na América Latina. Cad Saúde Pública 2003; 19 Suppl 1:S4-5.

30. Batista Filho M, Rissin A. A transição nutricional no Brasil: tendências regionais e temporais. Cad Saúde Pública 2003; 19 Suppl 1:S181-91.

31. Niewenweg R, Smit ML, Walenkamp MJE, Wit JM. Adult height corrected for shrinking and secular trend. Ann Hum Biol 2003; 30:563-9.

Recebido em 23/Jun/2011

Versão final reapresentada em 29/Fev/2012

Aprovado em 27/Mar/2012 